\title{
ASSISTÊNCIA AO BINÔMIO PACIENTE/FAMÍLIA NA SITUAÇÃO DE LESÃO TRAUMÁTICA DA MEDULA ESPINHAL
}

Ana Cristina Mancussi*

MANCUSSI, A.C. Assistência ao binômio paciente/família na situação de lesão traumática da medula espinhal.

Rev.latino-am.enfermagem, Ribeirão Preto, v. 6, n. 4, p. 67-73, outubro 1998.

Desenvolver pesquisas sobre a atuação do enfermeiro em reabilitação, atendendo a clientela específica tem sido muito gratificante mas um desafio, na medida em que a reabilitação é área emergente, que veio desmistificar o enfoque de um tratamento tardio, ou seja, muito posterior ao trauma. Com os objetivos de identificar e analisar as intervenções de enfermagem direcionadas à família ou ao binômio pacientelfamilia, optamos por uma metodologia que possibilitasse expressar os cuidados que os enfermeiros preconizam às pessoas com lesão traumática da medula espinhal. Coletamos dados de registros de consultas de enfermagem, identificando 14 diagnósticos de enfermagem e 183 intervenções pertinentes a eles. Utilizando a Técnica Delphi, foram validadas 76 intervenções pertinentes a oito diagnósticos de enfermagem mais frequentes. Deste total de intervenções (76), há 20 intervenções prescritas à família ou ao binômio pacientelfamília. Acreditamos ser necessário manter um canal de comunicação com a família desde o momento do trauma inserindo esta unidade de suporte no processo de reabilitação.

UNITERMOS: familia, enfermagem reabilitação

\section{INTRODUÇÃO}

O aumento do contingente de deficientes físicos, especialmente o dos portadores de lesão raquimedular, é um fato alarmante no mundo atual. Na maioria dos casos essas lesões têm origem traumática sendo o ferimento por arma de fogo (FAF), acidente automobilístico e queda as causas externas mais frequentes no Brasil (SPOSITO et al., 1986; SANTOS, 1989; FARO, 1991). Estas autoras em suas pesquisas obtiveram resultados que apontam as vítimas deste traumatismo, paraplégicos e tetraplégicos, como predominantemente adultos jovens, com idade variando entre 18 e 35 anos e na proporção de 4 homens para 1 mulher.

SANTOS (1989) estudou os aspectos epidemiológicos do trauma raquimedular (TRM) e apontou a necessidade de preparo mais específico das enfermeiras para assistir a este tipo de população.

Segundo SPOSITO et al. (1986) os recursos modernos da medicina e o desenvolvimento de novas técnicas na assistência para esta clientela têm permitido que um número cada vez maior destes pacientes consiga superar a fase aguda do TRM.

MENDONÇA NETTO et al. (1986) afirmam que os dados sobre a incidência dos TRM são bastante esparsos, mas consideram que a redução da mortalidade dos indivíduos portadores de TRM, principalmente na fase aguda, deve-se ao aprimoramento das técnicas de assistência ventilatória.

Pesquisadores como SANTOS (1989); AMÂNCIO \& SETTANNI (1990); SPOSITO et al. (1986), acreditam que o tratamento dos TRM deve ter início no momento do acidente por meio da avaliação primária e do transporte adequado da vítima, considerandoas como as primeiras e importantes ações a serem desenvolvidas, podendo com isso, amenizar ou mesmo impedir o chamado segundo acidente, presente em 3 a $10 \%$ dos TRM.

Sabemos que os avanços na área médica, bem como a conscientização da importância na prevenção das complicações, têm proporcionado a esse indivíduo chances maiores de sobrevivência.

Paralelamente a este fato, cabe aos profissionais envolvidos com a problemática do TRM, almejar não apenas o prolongamento como também a qualidade de vida para estes indivíduos. E nesse momento destacamos o papel relevante e fundamental da família para assegurar a continuidade dos cuidados planejados.

Uma sequela irreversível, certamente, traz à vida de uma pessoa portadora de paraplegia ou tetraplegia, profundas modificações que atingem a si próprio, à sua família e à sociedade onde vive.

Diante desta situação, gostaríamos de registrar que vários enfermeiros têm se empenhado no

\footnotetext{
* Professor Doutor do Departamento de Enfermagem Médico-Cirúrgica da Escola de Enfermagem da Universidade de São Paulo
} 
desenvolvimento do assistir, ensinar e pesquisar nesta área e, dentre eles podemos citar os estudos de COMARÚ (1982); SANTOS (1984); SANTOS (1989); FARO, (1991).

É do nosso conhecimento que o enfermeiro, sem formação especializada, vem assistindo a estes indivíduos juntamente com outros profissionais, promovendo a adaptação destes a uma nova situação, já que pacientes com lesão medular, estão sendo internados em hospitais gerais e apenas alguns poucos em centros especializados.

Preocupa-nos como é desenvolvido e aplicado este saber do enfermeiro; como ele vem assistindo a esta clientela, quais ações ele propõe diante de certas situações.

É importante lembrar que o paciente que apresenta TRM carrega consigo toda uma problemática de reintegração pessoal, familiar e social somada às alterações pertinentes ao aspecto físico ou orgânico.

Vale salientar que o conteúdo tratado neste trabalho faz parte de um estudo mais abrangente, tese de doutoramento (FARO, 1995), na qual identificamos os diagnósticos de enfermagem, intervenções de enfermagem relativas aos diagnósticos mais frequentes dos pacientes que apresentam TRM e que estavam em programa de reabilitação.Acreditamos que tanto o diagnóstico quanto as intervenções de enfermagem formam um raciocínio dinâmico contínuo que deveriam fundamentar a prática.

$\mathrm{Na}$ maioria das vezes, após internação devido ao TRM, há um período de tempo variável, para que esses lesados medulares, vinculem-se a um programa de reabilitação.Pelos depoimentos de vários pacientes, percebemos que isso acontece em virtude do número limitado de centros especializados, bem como por problemas de ordem social e econômica destes indivíduos que permanecem em casa até que consigam vaga em algum centro de reabilitação.

Acreditamos que o período de tempo entre a hospitalização e o programa de reabilitação, com todas as dificuldades advindas do TRM, proporcione ao indivíduo uma reflexão sobre os acontecimentos que vem vivenciando. Sabemos que é uma pessoa que retornou ao seu lar em condições diferentes e que gradativamente irá adaptar-se a estas novas condições.

Outro aspecto relevante e fundamental na reabilitação de uma pessoa, é o envolvimento da família, como elemento integrante da equipe assistencial, no sentido de participar e dar continuidade ao processo que está sendo implementado. Desta forma, reforçamos que o envolvimento da família como elemento integrante da equipe assistencial, é imprescindível no processo de reabilitação implementado.

A pessoa que apresenta sequelas advindas do TRM, gerando deficiência e incapacidades apresenta desde a fase aguda do trauma, níveis variados de dependência de outras pessoas para realização de atividades como vestir/despir, alimentar-se, sentar, bem como para higiene, eliminação urinária e fecal. Esta dependência varia conforme o nível e o grau da lesão medular e, também, com o tempo decorrente do trauma (FARO, 1996).

Assim, tentando compreender o processo de adaptação a uma nova situação de vida, o lesado medular não é o único nesta busca; há familiares envolvidos neste processo e que merecem estar assistidos nas suas dúvidas, medos, ansiedade, enfim na retomada de vida.

Por acreditar na assistência extensiva à família propomos, neste trabalho, os seguintes objetivos:

- identificar os diagnósticos de enfermagem das pessoas com lesão traumática da medula espinhal, segundo a classificação da NANDA (North American Nursing Diagnosis Association);

- identificar e discutir as intervenções de enfermagem, dirigidas à família de lesados medulares,face aos diagnósticos de enfermagem levantados.

\section{MATERIAL E MÉTODOS}

\subsection{Campo de realização do estudo}

Esta pesquisa foi realizada em uma instituição governamental, na cidade de São Paulo, onde são desenvolvidos programas de reabilitação às pessoas que apresentam deficiência física ou sensorial incapacitante.

\subsection{Material}

Inicialmente fizemos um levantamento da totalidade de prontuários de pacientes com lesão traumática da medula espinhal que estavam em programa de reabilitação entre os anos de 1988 e 1992. Deste total foram utilizados aqueles prontuários que tinham o Histórico de Enfermagem e a Evolução de Enfermagem preenchidos, compondo a documentação do prontuário. Em momento posterior, a população foi constituída por profissionais que formaram o grupo de juízes, os quais procederam à validação das intervenções de enfermagem relativas aos diagnósticos de enfermagem mais frequentes.

\subsection{Procedimento para coleta de dados}

A partir dos registros feitos nos históricos de enfermagem, formulamos os diagnósticos de enfermagem pela presença das características definidoras tal como proposto pela NANDA, utilizando a Taxonomia I.

Após a identificação dos diagnósticos de 
enfermagem, fizemos o levantamento das respectivas intervenções de enfermagem propostas frente aos diagnósticos, as quais estavam registradas na Evolução de Enfermagem.

Terminada esta etapa da pesquisa, iniciamos o processo de validação seguindo as normas propostas pela Técnica Delphi para a seleção dos enfermeiros que compuseram o quadro de juízes.

Consideramos validado o produto final que compreende as intervenções de enfermagem preconizadas pelos especialistas ou juízes.

Neste trabalho estaremos apresentando e analisando as intervenções de enfermagem preconizadas pelos juízes, voltadas à família e/ou paciente/família. Para tanto um dos passos metodológicos foi utilizar a Técnica Delphi em três fases para a validação das intervenções de enfermagem (FARO,1997), descrita resumidamente a seguir.

Esta Técnica permite obter consenso de grupo a respeito de um determinado fenômeno. O grupo é composto por juízes, ou seja, profissionais efetivamente engajados na área onde está se desenvolvendo o estudo. Ainda com relação aos juízes ou especialistas WILLIAMS \& WEBB (1994) consideram Delphi uma técnica de confiabilidade de resultados em função do grau de especialistas, não havendo um número ideal de juízes, sendo que a composição do grupo varia de acordo com o fenômeno em estudo e dos critérios definidos pelo pesquisador, para a seleção destes especialistas.

A técnica Delphi requer um conjunto de questionários para a obtenção das opiniões dos juízes. $\mathrm{O}$ primeiro deles, de maneira geral diferencia-se dos demais por ser mais abrangente tendo o objetivo de gerar a lista dos itens que irão compor os questionários posteriores (SPÍNOLA, 1984). Ainda em algumas pesquisas, utilizase escalas de valores onde se fixam as respostas (tipo "Likert scale"). Nos demais questionários a construção ocorre no sentido de facilitar a compreensão do estudado pelos envolvidos; para tanto as questões são detalhadamente esclarecidas e se fazem acompanhar de comentários, críticas e opiniões. Nestes não se utiliza mais a fixação das respostas em escalas de valores (SPÍNOLA, 1984).

O produto final desejável de um trabalho que utiliza a técnica Delphi, é a obtenção da opinião convergente de vários juízes, sendo uma técnica de consenso de grupo. Quanto ao consenso de grupo WILLIAMS \& WEBB (1994) consideram o nível de consenso arbitrário, decidido antes da análise dos dados, proposto pelo pesquisador. Comumente é relacionado a um valor numérico.

Em nossa pesquisa (FARO, 1995,1997) consideramos consenso, quando obtivéssemos, no mínimo, $70 \%$ de concordância nas respostas dos juízes.

\section{RESULTADOS E COMENTÁRIOS}

Apresentaremos a seguir os resultados pertinentes aos diagnósticos de enfermagem identificados, bem como as intervenções de enfermagem dirigidas ao paciente e família ou somente à família.

\subsection{Diagnósticos de Enfermagem}

Do levantamento dos 38 prontuários foi possível identificar 14 categorias diagnósticas, sendo o número mínimo de diagnóstico por pacientes cinco e o máximo 11.

A Tabela 1 mostra que foram identificadas 14 categorias diagnósticas à partir dos dados coletados nos históricos de enfermagem.

Tabela 1 - Distribuição de pacientes segundo os diagnósticos de enfermagem identificados São Paulo, 1993

\begin{tabular}{l|c|c}
\hline DIAGNÓSTICO DE ENFERMAGEM & N & $\%$ \\
\hline 01. Mobilidade física prejudicada & 38 & 100,00 \\
02. Potencial para prejuizona integridade & 38 & 100,00 \\
da pele & & \\
03. Déficit de autocuidado: vestir-se ef & 34 & 89,47 \\
ou arnumar-se & & \\
04. Déficit de autocuidado: barnho e/ou & 33 & 86,84 \\
higiene & & \\
05. Incontinência urinária & 27 & 71,05 \\
06. Potencial de infeç̧ão & 26 & 68,42 \\
07. Déficit de autocuidado: alimentação & 25 & 65,78 \\
08. Obstipação percebida & 20 & 52,63 \\
09. Distúrbios no padrão de sono & 9 & 23,68 \\
10. Retenção urinária & 9 & 23,68 \\
11. Integridade da pele prejudicada & 7 & 18,42 \\
12. Disfunção sexual & 5 & 13,15 \\
13. Incontinência intestinal & 3 & 7,89 \\
14. Déficit no autocuidado: tóilette & 3 & 7,89 \\
\hline
\end{tabular}

Podemos observar na Tabela 1, que os resultados apontam a totalidade (38) dos pacientes apresentando mobilidade física prejudicada e potencial para prejuízo na integridade da pele.

Formulamos os diagnósticos de enfermagem à partir dos dados registrados nos históricos de enfermagem e estes foram formulados face à presença das características definidoras tal como proposto pela NANDA. Ainda, posteriormente, estes diagnósticos foram referendados por duas enfermeiras, sendo uma da área assistencial e outra de ensino universitário. Ambas utilizam os diagnósticos de enfermagem da NANDA, na sua prática diária em assistência, ensino e pesquisa.

Durante a consulta de enfermagem, a enfermeira 
da equipe de lesado medular entrevista e registra de maneira descritiva cada um dos tópicos como sono e repouso, integridade cutâneo-mucosa, alimentação, hidratação, eliminações (urinária, fecal, menstrual), mobilidade e vestuário, higiene e conforto, sexualidade.

\subsection{Intervenções de Enfermagem}

Para BULECHEK (1985), intervenção de enfermagem é a ação autonôma da enfermeira, baseada em regras científicas que são executadas para beneficiar o cliente, seguindo o caminho preconizado pelo diagnóstico de enfermagem com o estabelecimento de metas a serem alcançadas. Ainda BULECHEK \& McCLOSKEY (1987) definem intervenção de enfermagem como uma direção no cuidar que a enfermeira realiza em favor do cliente/paciente durante o tratamento, sendo uma função essencial deste profissional. Caracterizam, ainda, a intervenção de enfermagem como um fenômeno cuja referência é a enfermeira, enquanto que o diagnóstico de enfermagem tem como referência o paciente.

Com base nos preceitos descritos anteriormente, apresentaremos os resultados pertinentes às intervenções de enfermagem, buscando compreender a direção no cuidar que envolve familiares/família nas ações preconizadas.

A partir dos registros feitos na evolução de enfermagem, foi possível identificar 183 intervenções de enfermagem propostas frente aos oito diagnósticos mais frequentes $(\geq 25 \%)$ nesta população as quais foram agrupadas segundo o verbo de ação.

Ainda, deste levantamento inicial, as intervenções listadas apontam o predomínio de ações no sentindo de orientar e treinar, o que nos parece compatível com a formulação dos respectivos diagnósticos e com a problemática dos lesados medulares em programa de reabilitação.

Cabe esclarecer que estas ações eram direcionadas aos próprios pacientes isoladamente ou somente para o familiar o qual cuida do paciente.

A seguir descreveremos as intervenções voltadas ao binômio paciente/família ou somente família e por diagnóstico de enfermagem.

\section{Mobilidade física prejudicada (13 intervenções)}

\section{Orientar o paciente e familiar quanto: (5 intervenções)}

- aos cuidados com almofada d'agua.

- à manutenção dos lençóis limpos e esticados.

- à altura compatível entre cama e cadeira de rodas.

- ao tipo de colchão adequado.

- ao tipo de cadeira de rodas adequado para facilitar o autocuidado (laterais removíveis, apoio nos pés, encosto alto para as lesões cervicais).
Treinar o paciente e familiar quanto: (1 intervenção)

- à realização do “push-up”(descompressão isquiática).

2. Potencial para prejuízo na integridade da pele (16 intervenções)

Orientar e treinar o paciente e o familiar quanto: (5 intervenções)

- à observação diária da pele do pênis quando utilizar dispositivo para incontinência urinária ou coletor plástico de urina.

- aos cuidados higiênicos, evitando contato e resíduos de fezes e urina na pele.

- à alimentação rica em proteínas e vitaminas.

- aos primeiros sinais de úlcera de pressão.

- à observação diária da pele utilizando espelho.

3. Déficit de autocuidado: alimentação (4 intervenções)

Orientar o paciente e familiar quanto: (1 intervenção)

- à necessidade de alimentar-se à mesa.

4. Déficit de autocuidado: banho e/ou higiene (8 intervenções)

Treinar o paciente e familiar quanto: (1 intervenção) - ao procedimento de higiene corporal.

\section{Incontinência urinária (12 intervenções)}

Orientar o paciente e familiar quanto: (1 intervenção)

- às anotações diárias de eliminação urinária, durante o período de reeducação vesical.

\section{Potencial para infecção (7 intervenções)}

Orientar o familiar quanto: (1 intervenção)

- aos cuidados com úlcera de pressão.

Treinar o paciente e familiar quanto: (1 intervenção)

- à realização do autocateterismo vesical intermitente.

\section{Obstipação percebida (12 intervenções)}

Orientar o paciente e familiar quanto: (4 intervenções)

- à realização de manobras de esvaziamento intestinal.

- à evitar uso constante de laxantes.

- à padronização de horários para evacuar: de 30 a 40 minutos após as refeições.

- ao aumento da ingestão hídrica. 
Diante destas descrições de intervenções, podemos observar que dos oito diagnósticos de enfermagem, sete deles geraram intervenções de enfermagem à família e/ou ao binômio paciente/família.

Relembramos que foram validadas 76 intervenções de enfermagem ao final da fase 3 - Delphi e que deste total havia 20 intervenções assim distribuídas: 1 intervenção era voltada à família e outras 19 eram dirigidas ao paciente e família, sugerindo a necessidade de assistência extensiva à família ou, pelo menos, conjunta.

Quanto ao verbo da ação, 20 das intervenções estavam voltadas direta ou indiretamente à família; as ações de orientar foram prescritas em 17 delas, enquanto que somente treinar foi prescrito em três intervenções.

Discorrendo sobre a assistência de enfermagem ao lesado medular, autores nacionais, enfermeiras efetivamente engajadas na assistência ao lesado medular, apontam cuidados como mudança de decúbito periódicas e sistematizadas, descompressão isquiática ("push-up"), posicionamento no leito pautadas pelo orientar, treinar e supervisionar (SASAHARA, 1980; KUGA, 1992; SAKAMOTO, 1992).

No entanto, a intervenção voltada à orientação e/ou treinamento da família desde a fase aguda da lesão medular pós-trauma é, praticamente, esquecida mesmo que seja uma assistência à família no sentido de ensina-la a "cuidar" ou a compreender e participar do "autocuidado".

Em 1996, tivemos a oportunidade de publicar um livro, sendo que um de seus capítulos aborda a assistência à pessoa com lesão traumática da medula espinhal onde enfocamos o desajuste individual e familiar como um aspecto ainda a ser mais explorado.

Por acreditarmos que as questões psicossociais relativas à qualidade de vida, assistência multidisciplinar à família sejam merecedoras do mesmo empenho nas pesquisas como as questões da esfera física ou biológica é que temos realizado atendimento ambulatorial à família, bem como inserido este conteúdo em nossas discussões (FARO, 1996; RONCARATTI \& PEREIRA, 1996).

A reabilitação no trauma deve ser precoce, com o objetivo de evitar deficiências ou mesmo de agravar incapacidades e, se considerada como um processo de aprendizagem, cabe ao paciente, família e profissionais de saúde, compartilharem da responsabilidade pela reconstrução gradativa de uma vida muito diferente e, neste processo de aprendizagem os cuidados físicos serão os primeiros a serem aprendidos. Já o ajustamento será um contínuo por toda a vida. (FARO 1996).

Também, no atendimento à família do paciente com lesão medular pós-trauma, cumpre-nos compreender que o ajustamento depende de uma conscientização interior, envolvendo redimensionar valores pessoais, conflitos e atitudes não apenas de quem sofreu o trauma físico, mas, principalmente daqueles que, de uma maneira ou de outra, compõe a unidade de suporte da qual o lesado medular faz parte, ou seja, a família.

\section{CONCLUSÕES}

Neste trabalho propusemo-nos a identificar e discutir as intervenções de enfermagem dirigidas à família ou familiar do lesado medular.Assim, em função do material e método por nós adotado, permitiu que chegássemos as seguintes conclusões:

- à partir dos registros feitos pelas enfermeiras nos históricos de enfermagem, identificamos 14 categorias diagnósticas.

- com a utilização da Técnica Delphi em três fases, foram validadas 76 intervenções de enfermagem, sendo que deste total 20 delas estão voltadas direta e indiretamente a família ou para o binômio paciente/família; as ações de orientar foram prescritas em sete delas enquanto que somente treinar foi prescrito em três intervenções.

\section{RECOMENDAÇÕES}

Considerando a nossa preocupação com a assistência, ensino e pesquisa sobre a atuação do enfermeiro junto ao lesado medular, permitimo-nos expressar esta preocupação na forma de recomendações.

Assim como o lesado medular é cuidado por um grupo multidisciplinar desde o atendimento pré-hospitalar, acreditamos que a sua família ou unidade de suporte mereça ser abordada para assistência desde o trauma e reeducada quanto à prevenção do trauma raquimedular (TRM), bem como das complicações dele decorrentes.

Além dos cuidados físicos, ou seja, aprender a fazer ou conhecer mudanças de decúbito, posicionamento no leito, prevenção de úlcera de pressão, reeducação vesical e intestinal, adequação de vestuário, da higiene e da alimentação, entre tantos outros focos de atenção, cabe manter um canal de comunicação com a família ou familiar para que ele possa expressar sua dúvidas, medos, preocupações e, também, que possamos atender a este familiar individualmente ou mesmo encaminha-lo a um outro profissional que esteja atendendo o paciente. Enfim, a família percorre uma trajetória de negação, adaptação, barganha, inseridos em um esquema de busca pela necessidade de ser compreendida e de revisão dos seus valores. Sob a ótica da pesquisa, são necessários outros estudos que possam compreender o evento de elaborar diagnósticos de enfermagem ao paciente que geram intervenções, as quais englobam a família. 

THE SPINAL MARROW

To develop research about nurse's action in rehabilitation, considering specific patients is very rewarding, but it is a challenge as rehabilitation is an emergent area that comes to abolish the focus of the late treatment, that is later than trauma. In order to identify and analyse the nursing interventions to the family, the author chose a methodology that can express the care nurses deliver to patients with spinal cord injury. The author collected data on nursing consultations identifying 14 nursing diagnoses and 183 interventions. Using the Delphi technique, 76 interventions were validated regarding to 8 nursing diagnoses that are more frequent. With reference to all interventions (76), there are 20 prescribed interventions to the family. We believe that it is necessary to keep a communication chanel with the family since the moment of trauma, inserting this support unit in the rehabilitation process.

KEY WORDS: nursing, family, rehabilitation

\section{ASISTENCIA AL BINOMIO PACIENTE/FAMILIA EN LA SITUACIÓN DE LESIÓN TRAUMÁTICA DE LA MEDULA ESPINAL}

Desarrollar investigaciones sobre la actuación del enfermero en rehabilitación atendiendo a la clientela especifica, viene siendo muy gratificante aunque un desafio, porque la rehabilitación es un área emergente, que ha desmitificado el enfoque de un tratamiento tardio o sea, muy posterior al trauma. Con los objetivos de identificar y analizar las intervenciones de enfermería direccionadas para la família o el binomio paciente-familia, optamos por una metodología que posibilitase expresar los cuidados que los enfermeros preconizam para las personas con lesión traumática de la medula espinal. Recolectamos los datos de los registros de las consultas de enfermería, identificando 14 diagnósticos de enfermería y 183 intervenciones pertinentes a ellos. Utilizando la Técnica Delphi fueron validadas 76 intervenciones pertinentes a 8 diagnósticos de enfermería más frecuentes. De este total de intervenciones (76), hay 20 intervenciones prescritas para la familia. Creemos que es necesário mantener una vía de comunicación con la familia desde el momento en que el individuo se traumatiza e insertarlo en esta unidad de soporte, en el proceso de rehabilitación.

TÉRMINOS CLAVES: família, enfermeria, rehabilitación

\section{REFERÊNCIAS BIBLIOGRÁFICAS}

01. AMÂNCIO, E.I.; SETTANNI, F. Traumatismo raquimedular tóraco-lombar. Rev.Bras.Med., v. 47, n.7, p.290-300, 1990.

02. BULECHEK, G.M. Nursing interventions: treatments nursing diagnoses. Philadelphia: Saunders, 1985.

03. BULECHEK, G.M.; McCLOSKEY, J.C. Nursing interventions: what they are and how to choose them. Holistic Nurs. Pract, v. 1, n. 3, p. 36-44, 1987.

04. COMARÚ, M.N. Problemas identificados pela enfermeira em pessoas na situação de deficiência física. São Paulo, 1982. 157 p. Dissertação (Mestrado) - Escola de Enfermagem, Universidade de São Paulo.

05. FARO, A.C.M. e Estudo das alterações da função sexual em homens paraplégicos. São Paulo, 1991. 98 p. Dissertação (Mestrado) - Escola de Enfermagem, Universidade de São Paulo.
06. FARO, A.C.M. e Do diagnóstico à conduta de enfermagem: a trajetória do cuidar na reabilitação do lesado medular. São Paulo, 1995. 207 p. Tese (Doutoramento) - Escola de Enfermagem, Universidade de São Paulo.

07. FARO, A.C.M. e Assistência de enfermagem ao paciente com traumatismo raquimedular. In: VENTURA, M. de F. et al. Enfermagem ortopédica. São Paulo: Ícone, 1996. p.175-89.

08. FARO, A.C.M. e Técnica Delphi na validação das intervenções de enfermagem. Rev. Esc. Enfermagem USP, v. 31, n. 2, p. 259-73, ago. 1997.

09. KUGA, C.K. Assistência de enfermagem a pacientes com traumatismo da coluna vertebral dorsolombar. In: NAKAMAE, D.D. et al. Temas de enfermagem em traumatologia do aparelho locomotor, São Paulo: Instituto de Ortopedia e Traumatologia do Hospital das Clínicas da Faculdade de Medicina da USP, 1992. p. 49-50.

10. MENDONÇA NETTO, A.B.F. de et al. Traumatismos raquimedulares fechado. Arq. Bras. Neuroc., v. 5, p. 1-35, 1986. 
11. RONCARATTI, E.; PEREIRA, V.G. Relacionamento enfermeiro-paciente-família. In: VENTURA, M. de F. et al. Enfermagem ortopédica. São Paulo: Ícone, 1996. p. 197-202.

12. SAKAMOTO, S.K. Assistência de enfermagem ao paciente com traumatismo da coluna cervical. In: NAKAMAE, D.D. et al. Temas de enfermagem em traumatologia do aparelho locomotor. São Paulo: Instituto de Ortopedia e Traumatologia do Hospital das Clínicas da Faculdade de Medicina da USP, 1992. p. 41-8.

13. SANTOS, W.L.R. dos. Funções da enfermeira na equipe multiprofissional de reabilitação. São Paulo, 1984. 102p. Dissertação (Mestrado) Escola de Enfermagem, Universidade de São Paulo.

14. SANTOS, L.C.R. dos. Lesão traumática da medula espinhal: estudo retrospectivo de pacientes internados no Instituto de Ortopedia e Traumatologia do Hospital das Clínicas da Faculdade de Medicina da Universidade de São Paulo entre 1982-1987. São Paulo, 1989. 102 p. Dissertação (Mestrado) - Escola de Enfermagem, Universidade de São Paulo.
15. SASAHARA, R.M. Assistência de enfermagem ao paraplégico. In: NAKAJO, A.S.H. et al. Temas de enfermagem ortopédica e traumatológica.

2. ed. São Paulo: Instituto de Ortopedia e Traumatologia do Hospital das Clínicas da Faculdade de Medicina da USP, 1980. p. 50-5.

16. SPÍNOLA, A.W. de P. Delfos: proposta tecnológica alternativa. São Paulo: Faculdade de Saúde Pública da Universidade de São Paulo, 1984.

17. SPÓSITO, M.M.de M. et al. Paraplegia por lesão medular: estudo epidemiológico em pacientes atendidos para reabilitação. Rev. Paul. Med., v. 104, n. 4, p. 196-202, 1986.

18. WILLIANS, P.L.; WEBB, C. The Delphi technique: a methodological discussion. J.Adv.Nurs., v. 19, n. 1, p.180-6, 1994. 